\title{
La visite en France de Pierre le Grand en 1717 dans la presse francophone ${ }^{1}$
}

\author{
Sergueï Mézine \\ Université de Saratov \\ mezinsa@mail.ru
}

\section{Резюме}

Визит Петра I во Францию (21 апреля - 24 июня 1717 г.) - важное событие в истории русскофранцузских культурных и дипломатических связей. Пребывание в Париже оставило яркий след в биографии знаменитого русского монарха, дало заметный толчок развитию петровской темы во французской литературе и общественной мысли. Европейская франкоязычная пресса уделила серьезное внимание путешествию царя. Газетные сообщения наряду с официальным журналом путешествия и мемуарными свидетельствами являются ценным источником, позволяющим реконструировать основные события визита, а также его восприятие в европейском обществе, в первую очередь - во французском. В основу статьи положены материалы четырёх франкоязычных газет. Две из них «Gazette de France» и «Le Nouveau Mercure» - выходили в Париже, «Relations Veritables» издавалась в Брюсселе, а «Gazette d'Amsterdam» - в Голландии.

Mots clé

Pierre $\mathrm{I}^{\mathrm{er}}$; Russie ; presse francophone ; France; Hollande.

La visite de Pierre le Grand en France (du 21 avril au 24 juin $1717^{2}$ ) est un événement marquant de l'histoire des liens culturels et diplomatiques entre la France et la Russie. Le séjour à Paris du célèbre monarque russe est un des moments brillants de sa biographie, et a donné une impulsion significative au développement du thème de Pierre le Grand dans la littérature et la pensée sociale françaises ${ }^{3}$.

La presse européenne a accordé une grande importance à ce voyage ${ }^{4}$. Les articles de journaux sont donc des sources de premier ordre, qui s'ajoutent au journal officiel du voyage et aux Mémoires des témoins. Cet ensemble de sources permet de reconstituer les principaux événements de la visite du tsar, ainsi que leur perception dans la société européenne. On ne peut attendre des journalistes du temps une analyse profonde de ces événements, mais la vision générale donnée par la presse rend bien compte, avec spontanéité, de la perception que ses contemporains européens avaient du tsar russe.

\footnotetext{
${ }^{1}$ Ce travail a été réalisé avec le soutien de la Maison des Sciences de l'Homme (Paris). Nous remercions Emmanuel Waegemans pour son aide.

${ }^{2}$ Toutes les dates sont données en nouveau style.

${ }^{3}$ Voir : С. А. Мезин, Пётр I во Франции [Sergueï Mézine, Pierre I en France], Saint-Pétersbourg : Evropeïski Dom, 2015. Le lecteur trouvera dans le livre un aperçu des sources et de la bibliographie concernant la visite de Pierre le Grand en France.

${ }^{4}$ On trouve une analyse des articles consacrés à la visite dans la presse germanophone dans l'article suivant : Astrid Blome, «Die zweite Westeuropareise Peters I. im Spiegel der zeitgenossischen Presse », Zar Peter der Große. Die zweite große Reise nach Westeuropa, 1716-1717, sous la dir. de Dieter Alfter, Hameln : Niemeyer, 1999 ; voir aussi Astrid Blome, Das deutsche Rußlandbild im frühen 18. Jahrhundert. Untersuchungen zur zeitgenössischen Presseberichterstattung über Rußland unter Peter I., Forschungen zur osteuropäischen Geschichte, Band 57, Wiesbaden : Otto Harrassowitz Verlag, 2000.
} 
Nous analyserons le traitement de la visite de Pierre le Grand dans quatre journaux : deux publications parisiennes, La Gazette de France et Le Nouveau Mercure, un journal de Bruxelles, Les Relations véritables, et La Gazette d'Amsterdam en Hollande. Ces périodiques sont, à ma connaissance, les seuls qui ont évoqué la visite de Pierre le Grand en France. On trouve encore une information à ce propos dans un hebdomadaire publié à La Haye, Les Nouvelles littéraires, mais elle a un caractère anecdotique.

Le journal officiel La Gazette de France paraît alors une fois par semaine, le samedi, sous le titre de Gazette. Son rédacteur est Eusèbe Renaudot, un abbé de la congrégation de l'Oratoire, orientaliste réputé et membre de l'Académie française. Ce journal, présenté sous forme épistolaire, est soumis à une censure stricte imposée par le gouvernement. On y trouve une série de lettres rédigées de l'étranger, mais aussi quelques lettres « de Paris ${ }^{5}$. Les journaux néerlandais utilisent une présentation identique, ce qui nous permet de les analyser de la même façon.

Le journal Le Nouveau Mercure a été créé par François Buchet ${ }^{6}$, un ancien conseiller et secrétaire du roi. Il se distingue par la richesse et la diversité de son contenu : dans cette publication mensuelle ${ }^{7}$, plus proche par sa forme des revues d'aujourd'hui, "toutes les nouveautés du temps » dans les domaines de la littérature, de la culture, des sciences et de la politique sont soumises à la réflexion des journalistes et des lecteurs.

Le journal bruxellois Relations véritables, aujourd'hui désigné comme «l'ancêtre des grandes gazettes européennes ${ }^{8}$, paraît quant à lui deux fois par semaine et dresse un panorama complet des événements politiques, des guerres et des relations diplomatiques dans les capitales européennes. Dans ce journal à l'orientation résolument catholique, et dont le rédacteur, à partir de 1693, est Jean Le Pape, les nouvelles de Rome tiennent le premier rang. Celles de Paris arrivent avec un retard de quatre jours.

La Gazette d'Amsterdam est aussi un journal solide et pérenne ${ }^{9}$. En 1717, elle paraît tous les mardi et les vendredi sous le titre suivant : Amsterdam. Avec privilège de nos Seigneurs des Etats de Hollande et de West-Frise ${ }^{10}$. Son fondateur, Jean Tronchin ${ }^{11}$, cherche à couvrir tous les événements politiques : on y trouve des rapports détaillés des événements survenus dans les capitales européennes (avec, au premier plan, Londres et Paris) ; en annexe, le rédacteur publie des documents politiques. En général, les nouvelles de Paris parviennent au lecteur dans un délai d'une semaine.

Bien que les récits de la visite de Pierre le Grand en France diffèrent, dans ces différents journaux, notamment par leur longueur, les principaux événements sont racontés de manière similaire et à peu près dans les mêmes termes, ce qui suggère l'existence à Paris d'une source officielle commune. L'existence d'une telle source est corroborée par une lettre écrite par un page de chambre ayant accompagné le tsar, de Libois, et adressée probablement au régent : «Il m'est venu dans l'esprit, que vous pourriez donner un petit plat au peuple dans cette occasion $<\ldots>$ Comme je vois que les Hollandais et autres ont débité des extravaguances, j'ai

\footnotetext{
${ }^{5}$ Dictionnaire des journaux. 1600-1789, sous la dir. de Jean Sgard, Paris : Universitas, 1991, vol. 1-2, ici vol. 2, p. 443-449.

${ }^{6}$ Dictionnaire des journalistes. 1600-1789, sous la dir. de Jean Sgard, Oxford : Voltaire Foundation, 1999, vol. 1-2, ici vol. 2, p. 168.

${ }^{7}$ Dictionnaire des journaux, vol. 2, p. 850-852.

${ }^{8}$ Dictionnaire des journaux, vol. 2, p. 1079.

${ }^{9}$ Dictionnaire des journaux, vol. 1, p. 451-453.

${ }^{10}$ Dans la suite de l'article le journal est désigné sous le titre Amsterdam.

${ }^{11}$ Dictionnaire des journalistes, vol. 2, p. 964.
} 
cru que je devrais envoyer un crayon de tout ce qui s'est passé... Le gazetier fera quelque belle pièce d'eloquence sur ce canevas, et le menu peuple sera content ${ }^{12}$. Cependant, ce sont justement les différences de présentation et de choix des détails qui présentent un grand intérêt pour les recherches.

Jean Rousset de Missy est un des premiers à utiliser les sources journalistiques pour compléter la biographie de Pierre le Grand. Dans son ouvrage Mémoires du règne de Pierre le Grand, publié pour la première fois en 1725-1726 sous le pseudonyme "russe » d'Iwan Nestesuranoi, il décrit la visite de Pierre le Grand en France à partir du journal de Buchet. Il est vrai qu'il ignore les articles parus séparément en mai sous forme de livre, mais il reprend mot à mot toute la chronique de juin du Nouveau Mercure ${ }^{13}$. Ce journal est également une source largement citée dans l'article de Boris Lossky, «Le séjour de Pierre le Grand en France ${ }^{14}$, qui prend en compte les publications de mai et de juin. De même, quand Albert Lortholary décrit comment la visite du tsar a été perçue en France, il connaît aussi le contenu des périodiques, au moins à partir des travaux de Lossky, même s'il préfère utiliser les informations de la Gazette de la Régence de Jean Buvat ${ }^{15}$, qui rapportait les «potins » et rumeurs de la ville. Celles-ci, en effet, conviennent mieux à Lortholary qui se propose de démontrer que «le voyage aboutissait à un échec, et l'échec politique se doublait d'un échec personnel ${ }^{16}$. Enfin, l'ouvrage d'Emmanuel Waegemans Pierre le Grand en Belgique ${ }^{17}$ rend bien compte des articles du journal de Bruxelles Relations véritables.

L'intérêt que les journaux francophones portent au voyage des années 1716 et 1717 de Pierre le Grand est bien antérieur à son arrivée à Paris. La gazette Amsterdam, qui propose une large couverture des événements politiques dans toute l'Europe, ne se contente pas d'observer les mouvements du tsar de Russie et de sa femme en Europe occidentale, mais, dans son édition $\mathrm{du} 1^{\mathrm{er}}$ janvier 1717 , porte aussi un jugement selon lequel la visite de Pierre le Grand en Hollande a été « un événement des plus remarquables de l'année dernière et d'un bon augure pour l'avancement de la Paix du Nord ». Dans le même article, le tsar est désigné comme «le fondateur et législateur d'un nouvel Empire ${ }^{18}$, et le journaliste souligne son intérêt pour l'art et les sciences qui doivent contribuer au développement de ses sujets. On trouve par ailleurs dans la gazette d'Amsterdam, à côté des échos bienveillants à l'égard de Pierre le Grand, d'autres articles sur des événements politiques polémiques où le rôle du tsar russe semble plus qu'ambigu, comme la présence de troupes russes en Mecklembourg ou la possible participation du tsar à l'intrigue des jacobites, soutenue par l'ambassadeur de Suède, contre le roi d'Angleterre George $\mathrm{I}^{\mathrm{er}}$. Le journal publie néanmoins, en supplément, le texte des Mémoires de Fedor Vesselovski, secrétaire de l'ambassade russe à Londres : il y félicite le roi d'Angleterre d'avoir déjoué la conspiration et nie toute existence de liens entre Pierre le Grand et les jacobites ${ }^{19}$.

\footnotetext{
${ }^{12}$ Cité d'après : Alfred Rambaud, «Les Russes à Paris. Pierre le Grand (1717). Simples notes », Revue politique et littéraire. Revue bleue, t. LII, n 16,14 octobre 1893 , p. 498.

${ }^{13}$ Ivan Nestesuranoi, Mémoires du règne de Pierre le Grand... $2^{\mathrm{e}}$ éd., Amsterdam : Weinsteins et Smith, 1729, vol. 14, ici vol. 3, p. 659-682.

${ }^{14}$ Boris Lossky, «Le séjour de Pierre Grand en France », in Le monde slave, Paris : Félix Alcan, 1932, 8, p. $278-303$.

${ }^{15}$ Jean Buvat, Gazette de la Régence. 1715-1719, Paris : G. Charpantier, 1887.

${ }^{16}$ Albert Lortholary, Les "philosophes » du XVIII siècle et la Russie. Le mirage Russe en France au XVIII siècle, Paris : Boivin, 1951, p. 20.

${ }^{17}$ Э. Вагеманс, Петр Великий в Бельгии, Saint-Pétersbourg : Giperion, 2007.

${ }^{18}$ Amsterdam, $\mathrm{1}^{\mathrm{er}}$ janvier $1717, \mathrm{n}^{\circ} 1$.

${ }^{19}$ Amsterdam, 30 mars 1717, $\mathrm{n}^{\circ}$ XXVI.
} 
Début avril 1717, la gazette Amsterdam annonce la volonté de Pierre le Grand de voyager dans le sud des Pays-Bas, à Anvers et à Bruxelles, et affirme que le tsar a décidé de revenir ensuite à $A_{m s t e r d a m}^{20}$. A ce moment-là, en réalité, le tsar a déjà résolu de se rendre à Paris, mais les visites qui suivent restent un grand mystère. C'est seulement dans le numéro du 20 avril d'Amsterdam (soit la veille de l'arrivée du tsar sur le territoire français) que paraît cette nouvelle de Paris: "Le Czar de la Grande-Russie est attendu ici du Pays-Bas. Un Gentilhomme Ordinaire du Roi partit d'ici le 8, pour aller le recevoir vers la frontière ; avec un Maitre d'Hôtel, un Contrôleur de la Bouche, et plusieurs autres officiers, pour le faire servir aux dépens du Roi ${ }^{21}$. La question des dépenses de l'ambassade russe est plus d'une fois évoquée dans les pages du journal : l'article du numéro du 23 avril précise que le tsar va rester à Paris jusqu'à la fin du mois avec des frais de 2000 livres par jour, puis, le 30 avril, le journal annonce un séjour de trois mois, pour 3000 livres par jour. Évidemment, ces informations ne sont pas exactes, tout comme celles qui supposent l'arrivée, incognito, d'autres monarques dans la capitale française. Mais les rumeurs sont fondées sur des faits, comme la préparation de la résidence royale pour des invités importants et l'arrivée à Paris du duc d'Holstein-Ploen. Le 6 mai, selon l'article de la gazette d'Amsterdam, une foule immense s'était rassemblée dans les rues de Paris en direction de Saint-Denis, dans l'attente d'un hôte de marque : on dit que le régent, le duc d'Orléans, se rend à la porte de Saint-Denis à la rencontre du tsar ${ }^{22}$.

La nouvelle de son arrivée à Paris parvient aux lecteurs de la gazette d'Amsterdam seulement dix jours plus tard :

De Paris le 10 mai. Le Czar de la Grande-Russie arriva vendredi [7 mai - ndlr] en cette ville à 9 heures du soir. Il était dans une calèche à six chevaux, et venait de Beaumont, où il avait couché ; il fut escorté jusques à St Denis par un régiment de dragons. Plusieurs princes et seigneurs de la cour qui étaient allés au devant de S. M., la conduisirent au Vieux Louvre dans les appartements qui lui avaient été destinés et la garde était postée en différents endroits. Ce Monarque refusa tous les honneurs qu'on voulut lui rendre à son entrée. Dès qu'il eut mis pied à terre, il entra d'abord dans l'appartement, où on lui avait préparé un magnifique souper, compose de 800 plats tant en gras, maigre, que dessert; mais cela ne servit que pour ceux de sa suite ; S. M. Czarienne n’ayant voulu boire qu'un verre de bière. Après avoir examiné la beauté des appartements, il remonta en carrosse à 11 heures, et alla coucher à l'Hôtel de Lesdiguières, où il restera pendant son séjour en cette ville. M. le Régent a été ce matin lui rendre visite ${ }^{23}$.

Le journal bruxellois Relations Véritables reprend quasiment au mot près cette «nouvelle de Paris », mais en y ajoutant quelques détails significatifs : Pierre le Grand est appelé " tsar de la Moscovie », ce que désapprouve la diplomatie russe ; on raconte qu'il est arrivé à Paris à " dix heures du soir », afin que personne ne puisse le voir, et qu'aucune torche n'éclairait son chemin, et enfin le journaliste complète le récit du repas du tsar, qui avait refusé de manger et n'avait voulu boire qu'un peu de bière, « et un peu... d'eau de vie $»^{24}$.

Un rapport détaillé de la visite du tsar en France du 21 avril au 12 mai paraît dans la rubrique « nouvelles de Paris » de la gazette Amsterdam, le 14 mai $^{25}$.

De tous les journaux ayant donné des informations sur le séjour du tsar à Paris, ce sont les périodiques français qui sont les plus complets. Néanmoins, l'information parvient au lecteur

\footnotetext{
${ }^{20}$ Amsterdam, 9 avril 1717, $\mathrm{n}^{\circ} \mathrm{XXIX}$.

${ }^{21}$ Amsterdam, 20 avril 1717, $\mathrm{n}^{\circ}$ XXXII.

${ }^{22}$ Amsterdam, 14 mai 1717, $\mathrm{n}^{\circ}$ XXXIX.

${ }^{23}$ Amsterdam, 19 mai 1717, $\mathrm{n}^{\circ}$ XL.

${ }^{24}$ Relations Véritables, 14 mai 1717.

${ }^{25}$ Amsterdam, 21 mai 1717, $\mathrm{n}^{\circ}$ XLI.
} 
de manière assez inégale : la gazette officielle donne des nouvelles de chaque mouvement du tsar, mais très brièvement ; Le Nouveau Mercure propose une chronique détaillée de sa visite, mais à la fin du mois.

La Gazette officielle est la première à évoquer la visite de Pierre le Grand dans les villes du sud des Pays-Bas (Pays-Bas catholiques) ${ }^{26}$. Le 8 mai, le journal rapporte que le roi a fait une visite à l'hôtel de Lesdiguières, accompagné du duc du Maine et du gouverneur de Sa Majesté, le marquis de Villeroy. On y a préparé un magnifique feu d'artifice, puis le jeune roi a assisté à une représentation de danseurs et de funambules ${ }^{27}$. C'est seulement dans le numéro suivant que l'on trouve des informations qui expliquent que la venue du roi était liée à la transformation de l'hôtel en résidence du tsar russe. Dans le numéro du 15 mai de la Gazette (c'est-à-dire avec une légère avance sur les journaux des Pays-Bas), on trouve un article qui annonce la venue du tsar en France et à Paris et donne les détails de son séjour jusqu'au 12 mai inclus $^{28}$. Les termes employés dans la rédaction de cet article sont quasiment les mêmes que ceux employés par les journaux néerlandais, et en les comparant entre eux, on s'aperçoit que cette source n'apporte pas de nouveaux détails et qu'elle est même, par endroits, plus laconique. En effet, l'article de la Gazette aborde seulement le déroulement superficiel de la visite. Ce journal, en général, se borne à donner des informations sur les rencontres officielles et les visites des institutions royales. Les informations sur les activités du tsar russe sont loin d'y occuper la première place.

On peut ainsi faire le bilan de toutes les informations sur la visite du tsar données dans ce journal : le 14 mai, il fait une visite au duc d'Orléans et à sa femme ; le même jour, il visite l'Académie royale de peinture et de sculpture, la grande galerie du Louvre, et regarde la collection royale de plans-relief, puis il se promène dans le jardin des Tuileries. Le 16 mai, le tsar se rend à l'hôtel des Invalides, le 17, il sort de Paris pour se rendre à la résidence royale de Meudon, le 19, il va pour la seconde fois à l'Observatoire ${ }^{29}$. Le 22 mai, il rend visite à la duchesse de Berry au palais du Luxembourg, et le 23 mai au régent dans sa résidence de SaintCloud $^{30}$. Enfin, le 24 mai, il rend visite incognito au roi et reste à Versailles, ce dont on trouve un récit plus détaillé dans le journal. Le 27 mai, le tsar revient à Paris, le 28 il visite l'Hôtel de la Monnaie et la bibliothèque royale. Le journal rapporte aussi son voyage à Fontainebleau (le 30 mai), et à l'abbaye de Saint-Denis (le 2 juin) ${ }^{31}$. Dans le numéro suivant de la Gazette, le journaliste raconte le second voyage du tsar à Versailles (du 3 au 12 juin), pendant lequel est organisée une fête en son honneur, et ses visites des châteaux de Clagny, Marly et SaintGermain-en-Laye. Le 12 juin, il revient à Paris et visite pour la seconde fois l'Hôtel de la Monnaie. Le 14 juin, des visites de l'Imprimerie royale et du Collège des Quatre-Nations à la Sorbonne sont organisées pour lui. Le 16 juin, il se rend à la manufacture des Gobelins, puis assiste à un défilé militaire sur les Champs-Elysées ${ }^{32}$. Le 26 juin, la Gazette relate pour la dernière fois une visite du tsar à l'Observatoire (le 17 juin) et aux Académies royales (le 19 juin) et mentionne que le tsar a échangé des visites avec le roi et le régent. Le dernier article décrit le départ du tsar de la capitale française le 20 juin ${ }^{33}$. On ne trouve qu'un commentaire,

\footnotetext{
${ }^{26}$ Gazette, 16 avril 1717, $\mathrm{n}^{\circ} 16 ; 24$ avril 1717, no. 17 ; 1 mai $1717, \mathrm{n}^{\circ} 18$.

${ }^{27}$ Gazette, 8 mai $1717, \mathrm{n}^{\circ} 19$.

${ }^{28}$ Gazette, 15 mai $1717, \mathrm{n}^{\circ} 20$.

${ }^{29}$ Gazette, 22 mai $1717, \mathrm{n}^{\circ} 21$.

${ }^{30}$ Gazette, 29 mai 1717, $\mathrm{n}^{\circ} 22$.

${ }^{31}$ Gazette, 5 juin 1717, $\mathrm{n}^{\circ} 23$.

${ }^{32}$ Gazette, 12 juin $1717, \mathrm{n}^{\circ} 24$; 19 juin, $\mathrm{n}^{\circ} 25$.

${ }^{33}$ Gazette, 26 juin $1717, \mathrm{n}^{\circ} 26$.
} 
que l'on peut interpréter comme un éloge de l'hôte russe. Il se trouve dans le numéro du 22 mai : «Sa Majesté Czarienne s'occupe tous les jours à voir dans les endroits publics et chez les particuliers, tout ce qui peut mériter sa curiosité, et satisfaire le goût qu'il a pour les Sciences et les beaux Arts ${ }^{34}$. Cette phrase est reprise mot pour mot dans les journaux d'Amsterdam et de Bruxelles ${ }^{35}$.

Un journaliste apporte une attention particulièrement soutenue à la visite du tsar en France, c'est François Buchet, le rédacteur du Nouveau Mercure. Le premier article qui y fait allusion raconte la venue du roi à l'hôtel de Lesdiguières. Si on le compare au même article dans la Gazette, où il est publié le 6 mai, on s'aperçoit qu'il fournit plus d'informations : les précisions données par le journaliste sur le déplacement et la suite du roi ne laissent plus aucun doute sur les liens entre ce voyage et la visite du tsar russe à Paris. On apprend que l'hôtel de Lesdiguières, meublé avec magnificence, est « destiné pour le logement du Czar » ${ }^{36}$. Dès les premières pages du numéro de mai, on trouve également cette brève information : « Le 7 le Czar arriva à Paris à neuf heures et demie du soir, et alla coucher à l'Hôtel des Lesdiguières, n'ayant pas voulu rester au Vieux Louvre dans l'Appartement de la feue Reine, qui était superbe ${ }^{37}$.

François Buchet donne une description encore plus complète de la visite du tsar dans un supplément spécial au numéro de mai de son journal, sous le titre Abrégé de l'Histoire du Czar Peter Alexiewitz, avec une Relation de l'État présent de la Moscovie, et de ce qui s'est passé de plus considérable, depuis son arrivée en France jusqu'à ce jour (A Paris, chez Pierre Ribou et Grégoire Dupuis..., 1717). Il est publié le 31 mai. Le journaliste y annonce sa volonté de présenter " un tissue fidele des evenemens de son régne ». Cependant, il ne présente à son lecteur qu'une compilation des ouvrages européens sur le tsar russe, notamment le livre de John Perry The state of Russia under the present Czar..., ce qu'il reconnaît lui-même (London, 1716). La précipitation du journaliste français, au moment de préparer l'ouvrage, l'a conduit à de nombreuses erreurs, particulièrement dans les pages «historiques" (p. 1-170). Au contraire, la chronique du séjour du tsar en France est riche en éléments et détails intéressants, bien que l'on ne puisse s'assurer de l'exactitude des faits, comme nous le verrons plus loin.

L'ouvrage de Francois Buchet est dédié «Au très sérénissime et très puissant Grand Seigneur Czar Pierre Alexievitz », avec l'indication du titre du tsar en entier. L'éloge du tsar, présenté comme un héros venu emprunter en Europe «toutes les richesses, soit politiques, soit morales », est doublé d'une image positive de la France comme nation de civilisation et d ' « émulation ». Toutefois François Buchet souligne que l' « empire » que le tsar a commencé à fonder et à « dégrossir » n'a pas encore atteint la perfection souhaitée par son réformateur. On pourrait être conduit à croire que cette initiative d'une publication dédiée au tsar a été financée par la Russie, mais, justement dans ce numéro du journal, on trouve un avertissement qui prévient les lecteurs de l'augmentation du prix du journal pour le mois de juin, en raison des frais d'éditions importants du supplément de mai : il passe de 15 à 20 sols.

Le récit détaillé par Buchet de la visite du tsar est précédé d'une courte chronique de son voyage à partir de son départ de Copenhague le 26 octobre 1716 jusqu'à son arrivée en France le 21 avril. Le récit du séjour de Pierre aux Pays-Bas est proche, pour sa teneur, de l'article du journal Amsterdam.

C'est à partir de l'arrivée du tsar russe en France que le récit devient plus original et plus

\footnotetext{
${ }^{34}$ Gazette, 22 mai 1717, $\mathrm{n}^{\circ} 21$.

${ }^{35}$ Amsterdam, 28 mai 1717, $\mathrm{n}^{\circ}$ XLII ; Relations Véritables, 25 mai 1717.

${ }^{36}$ Le Nouveau Mercure, mai 1717, $\mathrm{n}^{\circ} 96$.

${ }^{37}$ Le Nouveau Mercure, mai 1717, $\mathrm{n}^{\circ} 97$.
} 
précis : Buchet raconte la décision du 20 avril sur les rencontres que ferait le tsar dans les villes de France ; ses séjours à Dunkerque (du 22 au 25 avril), où il observa les travaux de l'écluse dans les fortifications, à Calais (du 25 avril au 4 mai), où il fêta Pâques et visita la forteresse, à Boulogne (les 4 et 5 mai), à Abbeville (les 5 et 6 mai) et à Breteuil ; ainsi qu'à Beaumont, où il déjeune avant d'entrer dans Paris ${ }^{38}$.

Buchet rapporte un épisode marquant du voyage : de passage devant la ville de Beauvais, le tsar refuse d'y entrer pour déjeuner chez l'évêque. On lui fait alors remarquer qu'il ne pourrait se restaurer correctement ailleurs, ce à quoi il répond : "Je suis un soldat, et pourvu que je trouve du pain et de la bière, je suis content ${ }^{39}$. Ce trait rapporté marque nettement la différence entre le tsar russe et les monarques français du temps, qui attachent beaucoup d'importance à la table. Plus tard, on retrouve la même anecdote sous la plume d'Andreï Nartov, auteur russe de la seconde moitié du XVIII ${ }^{\mathrm{e}}$ siècle : «Vous ne pensez qu'aux délices de la table. Mais le soldat, lui, se contente de pain sec et d'eau. Et ici, il y a du pain blanc et du vin $»^{40}$.

Le tsar, cependant, ne refuse pas toujours de se délecter des rafraichissements que lui préparent des hôtes soucieux de l'étiquette et désireux de le servir avec un luxe prestigieux. Le maréchal de Tessé, dans ses Mémoires, rappelle que le régent ne voit pas de réels enjeux politiques dans sa relation avec Pierre le Grand, et qu'il essaie surtout de le divertir jusqu'à son départ, sans jamais rien conclure avec lui ${ }^{41}$. A Beaumont, où le maréchal rencontre le tsar russe, un fastueux déjeuner lui est préparé par les serviteurs du roi, sous la direction de son premier maître d'hôtel, le marquis de Livry. C'est là que l'attendent six carrosses royaux (chacun pourvu d'un attelage de six chevaux), avec lesquels le tsar et sa suite entrent dans Paris, accompagnés d'une escorte de quinze gardes.

Les détails que rapportent Buchet au sujet de l'arrivée de Pierre le Grand à Paris, le soir du 7 mai, ne peuvent provenir que d'un témoin oculaire. On apprend par exemple que le jour de son arrivée, toute la route de Saint-Denis à Paris est bordée de deux rangées de voitures de curieux. Cependant, seuls les spectateurs les plus insistants restent quand il commence à faire sombre dans la rue. Les rues Saint-Denis et Saint-Honoré, par lesquelles le cortège du tsar passe pour aller au Louvre, sont illuminées. Au Louvre, le tsar examine les appartements richement arrangés, et demande au maréchal de Tessé de l'accompagner à l'hôtel de Lesdiguières. Enfin Buchet complète l'anecdote de « la bière et l'eau de vie », rapportée par d'autres journalistes : il ajoute que le tsar, à qui de nombreux plats abondants sont présentés, « demand[e] un morceau de pain et des raves, goût[e] à 5 ou 6 sortes de vins, b[oit] deux gobelets de bière qu'il aime beaucoup ${ }^{42}$.

$\mathrm{Au}$ sujet des visites officielles, la chronique de Buchet se contente de mentionner que le tsar a été occupé les 8 et 10 mai. Lors de sa première rencontre avec le duc Philippe d'Orléans, le tsar aurait dit qu'il avait pris la décision de venir en France dès qu'il avait su qu'il était devenu le régent. Le 10 mai, le jeune Louis XV se rend à l'hôtel de Lesdiguières, et le tsar, venu à la rencontre de sa voiture, « embrasse le Roi tendrement, lui reprit la main et ne la quitta pas

\footnotetext{
${ }^{38}$ Abbregé de l'Histoire du Czar Peter Alexiewitz, avec une Relation de l'Etat présent de la Moscovie, et de ce qui s'est passée de plus considérable, depuis son arrivée en France jusqu’à ce jour, Paris : Pierre Ribou et Grégoire Dupuis, 1717, p. 176-178.

${ }^{39}$ Abbregé de l'Histoire du Czar Peter Alexiewitz, op. cit., p. 179.

$4^{\circ}$ « Петр Великий», Воспоминания. Дневниковые записи. Анекдоты [« Pierre le Grand ». Souvenirs, journaux intimes, anecdotes], sous la dir. d'E.V. Anissimov, Moscou : fondation Pouchkine, Tretia Volna, 1993, p. 312.

${ }^{41}$ Mémoires et lettres du maréchal de Tessé, Paris, Treuttel et Würtz, 1806, t. I-II, ici t. II, p. 316.

${ }^{42}$ Abbregé de l'Histoire du Czar Peter Alexiewitz, op. cit., p. 181.
} 
jusqu'à ce qu'il l'eut mis dans son fauteuil ». Contrairement aux règles de l'étiquette, le tsar lui-même, sans l'aide des courtisans, le conduit dans les escaliers jusqu'à son cabinet et pendant tout le temps de l'entretien lui montre tant de signes de respect et de tendre attention «qu'on eût peine à retenir des larmes » ${ }^{43}$. Le duc de Saint-Simon, peut-être, se souvient de ce récit quand il écrit, des années plus tard, dans ses Mémoires : "On fut étonné de voir le Czar prendre le Roi sous deux bras, le hausser à son niveau, l'embrasser ainsi en l'air, et le Roi à son âge, et qui ne pouvait pas être préparé, n'en avoir aucune frayeur ${ }^{44}$.

Le texte des chroniques de Buchet, riche en petits détails, prétend à l'exactitude, mais le journaliste se trompe quand il rapporte que le tsar commence sa visite de Paris le 10 mai, le jour même de sa rencontre avec le roi. En fait, le tsar visite l'Arsenal et les places parisiennes où l'on rénove alors les statues du roi, seulement le lendemain, le 11 mai ${ }^{45}$. Un autre détail vient confirmer cette idée : apparemment, alors qu'il visite pour la première fois la capitale française, c'est-à-dire le 9 mai, le tsar demande au duc d'Antin de lui faire parvenir des documents sur les attractions de Paris. "Deux heures après », le tsar aurait déjà eu en main son "guide touristique", et aurait été agréablement surpris de trouver les descriptions traduites en «langue Esclavone ». Le tsar, ravi, aurait dit «qu'il n'y avait qu'un François capable de cette politesse ${ }^{46}$. Les journaux de Bruxelles et d'Amsterdam font coïncider la réception de la description de Paris et le compliment du tsar avec sa visite du palais du Luxembourg, le 22 mai $^{47}$. Mais le plus probable est que cette traduction ait demandé un certain temps et ait été donnée à Pierre le Grand deux semaines après son arrivée à Paris ${ }^{48}$. Buchet cependant considère la possibilité que cet événement ait eu lieu le premier jour de la visite.

L'auteur de la chronique du Nouveau Mercure insiste beaucoup sur la description des cérémonies de cour, il donne toujours la quantité exacte des voitures, indique les places qu'occupent le tsar, le roi et les courtisans, ainsi que la quantité des gardes qui les accompagnent. Dans les descriptions éloquentes du journaliste français transparaît sa fierté devant le lustre de la cour, la suprématie culturelle de la France, le savoir-vivre raffiné des courtisans, la richesse et la finesse de la langue française. L'auteur rapporte des compliments du tsar qui n'ont pas d'équivalent dans les sources russophones (ce qui, par ailleurs, ne veut pas forcément dire qu'ils sont moins proches de la vérité). Par exemple, pendant sa visite au roi, le 11 mai, Pierre le Grand aurait dit : « Je sais plusieurs langues, je voudrais les avoir toutes oubliées, et ne savoir que la Française, pour entretenir votre Majesté ${ }^{49}$.

François Buchet décrit les visites du tsar beaucoup plus précisément que le journal officiel : la visite de la manufacture des Gobelins le 12 mai, le jardin botanique royal, l'Observatoire et la manufacture de verre le jour suivant. Le 14 mai au matin, le tsar se rend à l'Académie royale de peinture et de sculpture, où Antoine Coypel lui explique les sujets des tableaux, puis

\footnotetext{
${ }^{43}$ Abbregé de l'Histoire du Czar Peter Alexiewitz, op. cit., p. 187-188.

${ }^{44}$ Saint-Simon, Mémoires, Moscou : izd. Progres, 1976, t. 1-2, ici t. 2, p. 331-332.

45 « Обстоятельный журнал о вояже (или о путешествии) его царского величества...», Гистория Свейской войны (Поденная записка Петра Великого), [ Journal circonstancié sur le voyage de Sa Majesté Impériale... », L'Histoire de la guerre suédoise (Journal de Pierre le Grand)], sous la dir. de T. S. Maykova, Moscou : Kroug, 2004, t. 1-2, ici t. 1, p. 616.

${ }^{46}$ Abbregé de l'Histoire du Czar Peter Alexiewitz, op. cit., p. 191.

${ }^{47}$ Relations Veritables, 28 mai 1717 ; Amsterdam, 1 juin 1717, $\mathrm{n}^{\circ}$ XLIV.

${ }^{48}$ On connaît deux manuscrits qui semblent directement liés à la visite parisienne de Pierre le Grand : «La description des choses curieuses de Paris » et « La description des maisons remarquables en France ». Voir : C.A. Мезин, « Роспись куриозным вещам в Париже : путеводители Петра I », Вестник РГНФ [Sergueї Mézine, «Liste des curiosités à Paris : les guides touristiques de Pierre I ${ }^{\mathrm{er}}$ », Bulletin de la RGNF], 2015, n³ (80), p. 52-62.

${ }^{49}$ Abbregé de l'Histoire du Czar Peter Alexiewitz, op. cit., p. 193.
} 
l'invité russe, dans la grande galerie du Louvre, regarde «les plans en relief des Places fortifiées du Royaume » et enfin se promène dans le jardin des Tuileries. Il se rend au Palais Royal du régent, où il est présenté à sa mère, Charlotte-Elisabeth de Bavière, avec qui il s'entretient en allemand. Le soir même, le tsar et le régent se rendent à l'opéra du Palais royal où l'on représente Hypermnestre. La description donnée par Buchet des agissements du tsar à la loge du Palais Royal est équivoque : selon lui, le tsar est « frappé " par la représentation et les danses de Mme le Prévôt, mais on sait aussi qu'il exprime bientôt le désir de se rafraîchir avec quelques verres de bière, et qu'il quitte le théâtre au beau milieu du quatrième acte ${ }^{50}$.

Le 16 mai, Pierre le Grand visite l'hôtel des Invalides et, d'après le témoignage du journaliste, exprime sa satisfaction devant l'existence d'un tel dispositif pour les vétérans. Le 17 mai, il se rend à la maison royale de Meudon, et, le lendemain, les diplomates russes et des envoyés d'autres gouvernements assistent à une audience chez le roi. Le 19 mai, le tsar va pour la seconde fois à l'Observatoire, où il brille par ses connaissances en astronomie.

Le long récit de la visite de Pierre le Grand à la duchesse de Berry au palais du Luxembourg n'est pas tant intéressant pour la grande quantité de détails donnés sur le cérémonial que pour l'information unique sur les goûts du tsar en peinture. A en juger par les articles variés qui témoignent de la visite de l'Académie royale de peinture, le tsar ne porte pas une attention particulière à l'art français de la Régence. Mais des œuvres du palais du Luxembourg lui plaisent beaucoup : le David de Guido Reni et Vénus demande à Vulcain de forger des armes pour son fils Enée d'Antoine Van Dyck, ainsi que la remarquable série de tableaux de PierrePaul Rubens qui raconte l'histoire de Marie de Médicis et d'Henri IV, et tout particulièrement la toile La naissance du dauphin ${ }^{51}$. Ces informations sur la visite à la duchesse de Berry sont datées du vendredi 21 mai, mais, comme le signale le journal de voyage, les 20 et 21 mai «Sa Majesté était à la maison et prit des médicaments », et le 22 mai, "Sa Majesté était chez Madame la duchesse du Berry $»^{52}$. Pour les journalistes la «légère indisposition » du tsar n'était pas non plus secrète: ils en parlèrent le 20 mai ${ }^{53}$. Grâce à l'aide des journaux néerlandais, la nouvelle de la maladie du tsar est vraisemblablement connue de Buchet, néanmoins il ne la diffuse pas. Il est possible que le récit de la visite au palais du Luxembourg ait été délibérément déplacé au 21 pour que le journal puisse évoquer, chaque jour du séjour du tsar, quelque information remarquable.

Le penchant du tsar russe pour les sciences et les arts apparaît clairement dans la chronique du rédacteur du Nouveau Mercure. Il témoigne de la visite du cabinet de curiosités du comte « Pajot d'Osambré » (Louis-Léon Pajot, comte d'Ons en Bray) à Bercy (le 22 mai), et aussi des rencontres entre le tsar et les grands maîtres des instruments mathématiques : Chapoteau, Byon, Buterfield. Selon Buchet, cela se reproduit trois ou quatre fois, et le tsar commande des instruments pour lui-même. Comme avant lui le journaliste du journal d'Amsterdam, le journaliste parisien remarque que le tsar s'entretient avec Buterfield en hollandais, sans traducteur ${ }^{54}$. Le journal mentionne même que lors de la visite du roi incognito aux Tuileries, le 24 mai, la conversation s'étant orientée sur le sujet d'une carte de la Moscovie, le tsar aurait évoqué sa tentative de relier la Volga et le Don par un canal. Cette information ne pourrait-elle pas avoir été influencée par la connaissance que Buchet avait du

\footnotetext{
${ }^{50}$ Abbregé de l'Histoire du Czar Peter Alexiewitz, op. cit., p. 196.

${ }^{51}$ Abbregé de l'Histoire du Czar Peter Alexiewitz, op. cit., p. 200-201.

${ }^{52}$ Journal détaillé du voyage de sa majesté le tsar, op. cit., p. 617.

${ }^{53}$ Relations Véritables, 25 mai 1717; Amsterdam, 28 mai 1717, ${ }^{\circ}$ XLIII.

${ }^{54}$ Amsterdam, 1 juin 1717, ${ }^{\circ}$ XLIV ; Abbregé de l'Histoire du Czar Peter Alexiewitz, op. cit., p. 202.
} 
livre de John Perry, qui a construit ce canal entre 1698 et 1701 ? Car la tentative s'était soldée par un échec, et le tsar n'avait pas de raisons d'aborder ce sujet. Ce même jour, le maréchal de Villeroy montre à Pierre le Grand les joyaux de la couronne. D'après Buchet, ce dernier en parle en connaisseur capable d'en juger la beauté et la valeur. Mais le duc de Saint-Simon semble plus proche de la vérité quand il raconte que le tsar, sur le sujet des joyaux, « dit qu'il ne s'y connaissait guère $»^{55}$.

D'autres déplacements du tsar, bien sûr, n'échappent pas à l'attention de l'auteur de la chronique : à la résidence du régent à Saint-Cloud et au château de Madrid dans le bois de Boulogne (le 23 mai), à Versailles et Marly (du 24 au 27 mai). C'est avec de vraies certitudes que le journaliste peut écrire que Pierre le Grand est transporté par les beautés de Versailles, où il navigue en barque sur le canal, fait des dessins et prend des notes dans son journal, et, à Marly, où il découvre le fonctionnement de la Machine. La garde royale le protégeait des regards des curieux dans les allées du parc.

De retour pour un bref séjour à Paris (du 27 au 29 mai), le tsar assiste à une cérémonie de fête dans la cathédrale Notre-Dame, visite l'hôtel de la Monnaie et la maison des Enfantstrouvés, et enfin prend du repos et se livre à des divertissements dans le château du duc d'Antin à Petit-Bourg et à Fontainebleau (le 30 mai). Buchet termine ainsi la chronique de mai, et y ajoute quelques descriptions telles que celles des principales figures de la suite et de la famille du tsar, et enfin un portrait de Pierre le Grand : "Le Czar âgé de 45 ans, est grand, d'une stature d'environ cinq pieds et demi, bien pris dans sa taille, n'étant ni maigre, ni gras, brun de visage, des yeux très vifs, le nez et la bouche bien ensemble, relevée de deux petites moustaches noires, avec une physionomie élevée et en même temps affable. Ses habits à la française sont des plus simples, le surtout qu'il porte ordinairement est brun, avec un petit bordé d'or ; sa perruque de cheveux presque noirs est en façon de celle d'un abbé, et il dîne entre 10 et 11 heures; soupe légèrement, se couche de bonne heure, et se lève de grand matin $»^{56}$. Saint-Simon a sûrement sous les yeux ce passage de Buchet, quand, dans ses célèbres Mémoires ${ }^{57}$, il décrit l'apparence de Pierre le Grand.

François Buchet considère qu'il doit poursuivre la chronique du séjour de Pierre le Grand en France dans le numéro de juin du Nouveau Mercure. Elle commence par un article qui raconte la participation du tsar à une chasse aux cerfs dans la forêt de Fontainebleau. Le comte de Toulouse lui organise une partie de ce divertissement royal, auquel le tsar prend part la première fois. D'après le journaliste, le tsar est très satisfait de la chasse, qui dure une heure et demie. Encore une fois, le récit de Saint-Simon semble plus juste : il dit que la chasse ne plaît pas à Pierre, qui, en général, n'aime pas ce divertissement ${ }^{58}$. Contrairement aux attentes de son hôte, Pierre quitte Fontainebleau le jour même.

Le premier juin, le tsar et sa suite naviguent de Petit-Bourg à Choisy, où ils sont reçus par la princesse de Conti et visitent sa résidence. Le tsar passe en péniche sous tous les ponts de Paris jusqu'à la Porte de Conférence, où il prend une voiture pour passer les remparts de la ville. Il se procure ensuite dans une boutique de pyrotechnie une grande quantité de pétards et de fusées qu'il lance lui-même dans le jardin de l'hôtel de Lesdiguières.

Plus loin, le journaliste raconte le voyage du tsar le 2 juin à l'abbaye de Saint-Denis. Il ne se contente pas de décrire les activités du tsar - contempler l'église, le trésor, les tombes royales et les nouvelles constructions - mais il intervient aussi en tant que narrateur interne, avec des effets de style, quand il souligne que le tsar a été impressionné par la puissance des murs et

\footnotetext{
${ }^{55}$ Abbregé de l'Histoire du Czar Peter Alexiewitz, op. cit., p. 204 ; Saint-Simon, Mémoires, op. cit., t. 2 , p. 336.

${ }^{56}$ Abbregé de l'Histoire du Czar Peter Alexiewitz..., op. cit., p. 208.

${ }^{57}$ Saint-Simon, Mémoires, op. cit., t. 2, p. 329-330.

${ }^{58}$ Le Nouveau Mercure, juin 1717, 183 ; Saint-Simon, Mémoires, op. cit., t. 2, p. 338.
} 
des voûtes, et que des cellules, où les bénédictins lui préparent un festin, s'étend une vue merveilleuse. Sur le chemin du retour pour Paris, le tsar est reçu par le duc de Tresmes à SaintOuen.

Le 3 juin, accompagné de quelques membres de la noblesse française, le tsar passe quelques jours à Versailles. Pierre le Grand et sa suite s'établissent au Trianon, d'où ils organisent de petites excursions dans les environs. Le 6 juin il visite le château de Clagny, va voir le grand aqueduc, puis se rend à Marly, où il s'intéresse aux fontaines et aux cascades, particulièrement à la cascade d'Agrippine. La fête donnée dans le jardin de Marly le 10 juin est le point culminant du séjour : feux d'artifice, illuminations, musique et danses se prolongent jusque tard dans la nuit, et le lendemain, contrairement à ses habitudes, le tsar se lève tard. Le journaliste ne précise pas que la fête a été donnée pour l'anniversaire de Pierre, mais il a tout à fait raison quand il souligne que le tsar a été très content « de cette galanterie ». Il décrit, dans une lettre à sa femme, son séjour à Versailles comme « un grand plaisir » ${ }^{59}$.

Le tsar quitte Versailles le 11 juin pour se rendre à Saint-Germain-en-Laye, et ensuite à Saint-Cyr, où Madame de Maintenon a fondé un pensionnat pour jeunes filles et où ellemême achevait alors sa vie. Le journaliste raconte que l'école plaît beaucoup au tsar, et d'autres contemporains ont également rapporté que le tsar a été intéressé par la personne de la favorite du roi soleil, cette curiosité du "grand siècle », " qui reçût sa visite sur son lit " ${ }^{60}$. Le tsar ne quitte Versailles que le 12 juin après avoir revu une dernière fois les appartements et les collections du grand palais.

Sur la route du retour de Versailles il rend visite, à Chaillot, à la veuve du roi d'Angleterre, puis il va au Louvre, où l'on a fait graver, à l'Hôtel de la Monnaie, une médaille en son honneur. On lui donne aussi un portrait de lui réalisé par l'artiste Jean Duvivier : il est semblable à l'image de la médaille, mais gravé en relief sur du bronze doré.

La chronique du Nouveau Mercure témoigne ensuite qu'après quelques rencontres diplomatiques (avec le nonce apostolique et les ambassadeurs du Portugal et de Malte le 13 et 14 juin), le tsar poursuit ses excursions «d'apprentissage ». Le 14 juin (dans le journal on donne deux fois, par erreur, la date du 15), il se rend à l'Imprimerie royale et au Collège des Quatre-nations, où le célèbre géomètre Pierre Varignon lui donne une leçon. Le journaliste rappelle que le monarque russe a déjà établi des écoles similaires à Moscou et SaintPétersbourg (il pensait probablement à l'école de navigation et à l'Académie de la marine) et qu'il avait le projet d'en fonder de nouvelles ${ }^{61}$. Après avoir acheté sur la route une sphère mouvante selon le système de Copernic au maître Pigeon, le tsar se rend à la Sorbonne, où il rencontre les professeurs et observe le mausolée du cardinal de Richelieu. Il gagne ensuite l'île de la Cité et il monte dans la tour de la cathédrale Notre-Dame, d'où il peut contempler Paris vu du ciel.

Le 15 juin, le tsar se rend à la manufacture royale des Gobelins, et après le déjeuner il se promène dans Paris en calèche et s'arrête dans les maisons des aristocrates français. Le journal le Nouveau Mercure enrichit la description des troupes assemblées sur les ChampsÉlysées pour le tsar, le 16 juin $^{62}$ : il explique qu'à cause de la poussière soulevée ce dernier a

\footnotetext{
59 Le Nouveau Mercure, juin 1717, 188 ; Переписка русских государей и других особ царского семейства [Correspondance des souverains russes et des personnes de la famille du tsar], t. 1-5, Переписка Петра I c Екатериною Алексеевною [Correspondance de Pierre $I^{\text {er }}$ avec Catherine Alexeevna], Moscou : Orlov, 1861, vol. I, p. 71.

${ }^{60}$ Le Nouveau Mercure, juin 1717, p. 188 ; Saint-Simon, Mémoires, t. 2, p. 339.

${ }^{61}$ Le Nouveau Mercure, juin 1717, p. 191-192.

${ }^{62}$ Le Nouveau Mercure, juin 1717, p. 194-195.
} 
rapidement quitté la parade, puis qu'il s'est entretenu pendant une heure et demie avec le duc Philippe d'Orléans (par l'intermédiaire du prince Kourakine), et s'est promené dans le jardin des Tuileries. Les 16 et 17 juin le tsar poursuit ses visites à la noblesse parisienne : il déjeune chez le gouverneur de Paris, le duc de Tresmes, et chez le maréchal de Villars.

A en juger par les articles du journal, les derniers jours du séjour de Pierre à Paris sont principalement consacrés à satisfaire son intérêt pour les sciences. Ainsi, le 18 juin, il s'entretient longuement avec le géographe Guillaume Delisle, notamment au sujet de deux cartes manuscrites représentant les régions orientales de son empire, peuplées de Tatares et de Kalmoukes; puis le chimiste Geoffroy fait une démonstration de ses expériences de chimie. Le même jour, le tsar et Philippe d'Orléans échangent leurs visites d'adieu et le tsar se rend incognito chez le roi. Il lui offre comme cadeau d'adieu un plan de Saint-Pétersbourg. A en juger par les caractéristiques données : « on peut juger de l'importance et de la grandeur de cette Place, par les 32 Bastions qui doivent la défendre» ${ }^{63}$, la conversation évoque le projet global établi par Jean-Baptiste-Alexandre Le Blond début 1717, que Pierre le Grand a reçu aux Pays-Bas la veille de son départ pour la France. D'après un des historiens de SaintPétersbourg, « ce projet lui était nécessaire [à Pierre le Grand - ndlr] pour faire parler de lui en Europe occidentale $»{ }^{64}$. Enfin, le tsar et son médecin personnel Areskine invitent le médecin anglais Woolhouse à l'hôtel de Lesdiguières. Il réalise devant le monarque une opération de l'œil d'un vétéran : « Le Czar voyant planter l'aiguille dans l'œil de l'invalide, se détourna un moment : mais la curiosité l'ayant emporté, il le vit travailler et eût la preuve que la cataracte était abattue ${ }^{65}$. Ayant constaté le succès de l'opération, le tsar demande au médecin de l'enseigner aux étudiants russes.

Un petit détail n'échappe pas à l'attention du journaliste : le 19 juin, dès le matin, le tsar se rend aux bains installés pour lui, qu'il utilise habituellement le samedi. Puis on arrange un déplacement au Parlement parisien. Après le déjeuner, le jeune roi se présente pour une visite d'adieu à l'hôtel de Lesdiguières. Le même jour, Pierre le Grand fait une visite importante à l'Académie royale des sciences. Il approuve les réalisations présentées par les scientifiques : «S. M. Cz. a prêté beaucoup d'attention à toutes les nouveautés, et a bien voulu prendre séance, permettant à la compagnie de s'asseoir... " ${ }^{66}$. Ensuite, à l'Académie des inscriptions, on montre au tsar l'histoire du roi Louis XIV en médailles. La capacité des Français à célébrer leurs monarques impressionne visiblement Pierre : le 20 juin, avant son départ, il retourne à l'Hôtel de la Monnaie pour revoir la médaille dédiée au Roi Soleil.

Le 20 juin au soir, après le service religieux à l'hôtel de Lesdiguières (c'était le jour de la Sainte Trinité), Pierre le Grand quitte Paris, accompagné d'une escorte de mousquetaires du roi jusqu'à Soissons ${ }^{67}$. Au dîner, à Livry, le prestigieux hôte boit à la santé du roi et du régent.

Avant son départ, le tsar récompense généreusement tous les hauts fonctionnaires responsables de lui pendant son voyage, ainsi que les serviteurs du roi qui lui ont assuré des conditions de séjour confortables à Paris : le duc d'Antin, les maréchaux de Tessé et d'Estrées, le marquis de Livry et le maître d'hôtel royal de Verton reçoivent des portraits miniatures du tsar ornés de diamants, d'autres reçoivent des montres en or, des médailles souvenir et de

\footnotetext{
${ }^{63}$ Le Nouveau Mercure, juin 1717, p. 198.

64 Т.А. Базарова, Планы петровского Петербурга: Источниковедческое исследование [T.A. Bazarova, Les plans de St.-Pétersbourg du temps de Pierre le Grand : Etude des sources], St.-Pétersbourg : Naouka, 2003, p. 108.

${ }^{65}$ Le Nouveau Mercure, juin 1717, p. 199.

${ }^{66}$ Le Nouveau Mercure, juin 1717, p. 202.

${ }^{67}$ Sans faire allusion à la visite du tsar, le journal Amsterdam publie que le 20 juin, à Paris et dans les environs, se sont levés « un vent impétueux, avec une grosse gréle et une pluie violente qui ont fait de grands ravages tant en cette ville qu'aux environs ». Amsterdam, 6 juillet 1717, $\mathrm{n}^{\circ} \mathrm{LIV}$.
} 
l'argent ${ }^{68}$.

Après quelques arrêts à Livry, Soissons, Arétale, et une visite de Reims, le tsar atteint la frontière des Pays-Bas et part le long de la Meuse pour Liège.

Les journaux des Pays-Bas, qui relâchent leur attention dès l'arrivée de Pierre le Grand à Paris, publient beaucoup de nouvelles de ses autres séjours en France. Ces écrits sont plus courts que la chronique de Buchet, mais ils sortent plus tôt, et donnent donc des nouvelles plus fraîches. Les articles des journaux français et néerlandais se ressemblent beaucoup, mais une étude précise permet de relever les divergences et les ajouts d'informations supplémentaires.

Par exemple, les Relations véritables ne décrivent pas de la même façon la première excursion de Pierre le Grand à Paris le 11 mai : pour ce journal, la visite de la ville commence par le Pont-Neuf, sur lequel on a élevé une statue d'Henri IV, et se poursuit par une visite de la Samaritaine "nouvellement rétablie " ${ }^{69}$. C'est peut-être parce que le journaliste bruxellois connaît l'intérêt réel du monarque russe pour les sculptures royales, qu'il ajoute au nombre des monuments montrés au tsar le plus vieux monument équestre de Paris et les fontaines de la ville décorées de sculptures. Ce journal date par erreur la visite de l'hôtel des Invalides du 12 mai, mais dès le numéro suivant on trouve un rectificatif $7^{70}$. Il se distingue de François Buchet qui écrit que le tsar va aux bains le samedi en affirmant que le monarque « prend le bain tous les jours une fois $»^{71}$.

D'autres points de divergences concernent les descriptifs des monuments de Paris proposés au tsar en russe, ou encore de petits détails dans le récit des visites de l'Hôtel de la Monnaie, de Versailles, de Fontainebleau. Les journaux néerlandais indiquent quelques événements oubliés par l'éditeur méticuleux du Nouveau Mercure, par exemple la participation du tsar à une fête religieuse (jour de l'Octave de la Fête Dieu) le 3 juin : « Sa Majesté Czarienne fut sur un balcon dans la rue Saint-Antoine, pour voir passer la procession de St Paul $»^{72}$, ainsi que la visite d'un certain « Mr Hogguer», rue Saint-Martin, pour voir le « petit vaisseau » (il s'agit vraisemblablement d'un modèle de bateau) ${ }^{73}$.

La gazette d'Amsterdam donne surtout un grand nombre de détails intéressants sur la vie du tsar à Paris : « Il est toujours servi à l'Hôtel de Lesdiguières par les officiers du Roi, qui lui préparent son diner pour 10 heures ; il ne soupe presque pas, et se couche de bonne heure. Sa table est de 14 couverts ; et il n'a environ que 30 personnes à sa suite. Il a fait habiller ses gens à la Française, d'une étoffe verte avec un gallon d'or sur l'habit et la veste » ${ }^{74}$. Si François Buchet, dans son journal, ne mentionne que les achats d'instruments mathématiques faits par le tsar, la gazette d'Amsterdam apporte des informations supplémentaires : les 13 et 14 juin, « S. M. Cz. fut voir les beaux magasins du Sieur Calley, marchand près du Pont-Neuf. On dit qu'Elle y a acheté de beaux meubles et beaucoup de curiosités ${ }^{75}$. En effet, on sait que le tsar a dépensé beaucoup d'argent à Paris pour acheter des meubles, des textiles, des vêtements et des objets de luxe, ainsi que des livres ${ }^{76}$.

\footnotetext{
${ }^{68}$ Le Nouveau Mercure, juin 1717, p. 204-205.

${ }^{69}$ Relations Véritables, 18 mai 1717.

${ }^{70}$ Relations Véritables, 18 mai 1717 ; 25 mai 1717.

${ }^{71}$ Relations Véritables, 28 mai 1717.

${ }^{72}$ Relations Véritables, 18 mai 1717.

${ }^{73}$ Amsterdam, 25 juin 1717, $\mathrm{n}^{\circ} \mathrm{LI}$.

${ }^{74}$ Amsterdam, 25 mai 1717, $\mathrm{n}^{\circ}$ XLII.

${ }^{75}$ Amsterdam, 25 juin 1717, $\mathrm{n}^{\circ} \mathrm{LI}$.

${ }^{76}$ Voir Сборник выписок из архивных бумаг о Петре Великом [Recueil de notes des papiers d'archives sur 
L'article sur la visite du tsar inséré dans le journal de La Haye Les Nouvelles littéraires, dirigée par Henri du Sauzet, revêt un caractère anecdotique. Il s'agit de l'examen, par Pierre, « du cabinet et [de] tous les instrumens de mécanique du P. Sebastien, carme de la place Mobert ${ }^{77}$. Le numéro précédent des Nouvelles littéraires avait publié une lettre d'un lecteur du journal de Paris Le Nouveau Mercure. Il déplorait l'absence de toute description de cette visite dans le journal parisien. L'éditeur du journal de La Haye a donc tenu à raconter à ses lecteurs avec quelle méticulosité et quel savoir le tsar russe avait examiné le cabinet du père Sébastien. L'auteur émaille sa description de détails curieux: après l'observation des instruments, le tsar s'est jeté avidement sur le repas proposé par l'hôte de la maison, s'est luimême coupé du pain et a demandé une grande coupe de vin, qu'il a bue d'un trait, puis a proposé à l'hôte de boire dans la même coupe. La visite du tsar chez un moine savant est prouvée par une lettre de ce dernier adressée au médecin du $\operatorname{tsar}^{78}$, cependant la date exacte de cette rencontre reste incertaine. La date communiquée dans le journal hollandais, le samedi 17 avril, ne correspond pas à la réalité : le 17 avril le tsar n'était pas encore à Paris, de plus ce n'est pas un samedi. On pourrait se demander si toute cette histoire n’a pas été inventée par le journaliste de La Haye afin de montrer le comportement « barbare » du tsar.

\section{Conclusion}

Pour conclure cette étude sur les journaux francophones comme sources d'informations sur la visite de Pierre le Grand en France, il nous faut une fois encore souligner les spécificités des périodiques que nous avons examinés. La Gazette de France diffuse des nouvelles brèves, des rapports purement officiels. La liste des déplacements du tsar est loin d'être complète : il ne s'agit que des rencontres officielles, des visites des institutions et des résidences royales. Ces articles ne caractérisent jamais le tsar, à l'exception de remarques laconiques sur son intérêt pour les sciences et les arts. Par ailleurs, les activités diplomatiques du tsar sont restées inconnues des journalistes, bien que les journaux aient recensé un certain nombre de contacts officiels. L'entretien avec le régent, les discussions des diplomates, la préparation de l'accord d'union franco-russe restent le plus strict secret. Le journal Amsterdam remarque bien une grande affluence de diplomates étrangers dans la capitale hollandaise le 15 août $1717^{79}$, soit le jour de la signature de l'union d'Amsterdam entre la France, la Russie et la Prusse, mais le fait même de l'union n'est pas relevé dans la presse. Les éléments fournis par les Relations véritables bruxelloises couvrent plus largement le séjour du tsar en France, bien que les journalistes ne fassent pas preuve de beaucoup d'originalité. Dans la gazette Amsterdam, on perçoit un plus grand intérêt pour ce sujet. Pour son rédacteur, cette visite fait partie intégrante des voyages du tsar et doit être considérée à l'aune du contexte politique européen. Le journaliste d'Amsterdam s'intéresse ainsi à un nombre de questions plus large, allant du problème des pourparlers de paix dans le Nord de l'Europe aux détails de la vie quotidienne de Pierre le Grand à Paris. Enfin, le Nouveau Mercure consacre des dizaines de pages exclusivement à Pierre le Grand et à son séjour en France. C'est là qu'on trouve la chronique la plus complète de la visite, développée dans une tonalité bienveillante et même flatteuse. De toute évidence, le rédacteur, Buchet, revendique son intérêt pour «toutes les nouvelles » pouvant être diffusées au sujet du tsar russe réformateur, qui change et modernise la vie de ses

\footnotetext{
Pierre le Grand], Moscou : Tip. Ouniversiteta, 1872, t. 1-2, ici t. 2, p. 62-70, 81-82.

${ }^{77}$ Nouvelles littéraires. $1717, \mathrm{n}^{\circ}$ 5, p. 389-390.

${ }^{78}$ La filiale de Saint-Pétersbourg des Archives de l'Académie des sciences de Russie (SPF ARAN), fonds 1, op. 3, $\mathrm{n}^{\circ}$ $5, \mathrm{f}^{\circ} 57-58$.

${ }^{79}$ Amsterdam, 17 août $1717, \mathrm{n}^{\circ}$ LXVI.
} 
sujets. En quête d'exhaustivité, l'auteur cherche à représenter toutes les facettes du tsar, jusqu'à son discours, et il dépasse parfois les limites de l'authenticité, au point qu'il est difficile de prouver ou de réfuter toutes ses informations. Parallèlement, sa description du voyage se transforme parfois en une sorte de « guide de voyage » de Paris et de ses environs, ce qui manque un peu d'originalité. Tous les textes de ces journaux utilisent les clichés caractéristiques de la présentation des monarques dans la presse, ce qu'Albert Lortholary appelait « la banalité officielle ». Quelques différences peuvent néanmoins être notées au sein de ce discours «politiquement correct» des journalistes : seules les éditions d'Amsterdam appellent la Russie et les Russes « Russie » et « Russiens », et font coïncider le titre de tsar et celui d'empereur. A cette période, les éditions de Paris et de Bruxelles utilisent encore les termes traditionnels de «Moscovie » et «Moscovites », auxquels s'opposent les diplomates russes.

Dans l'ensemble, la presse francophone contient les informations les plus complètes concernant l'aspect public de la visite de Pierre I en France. De ce point de vue, c'est une source plus précieuse que le court journal de voyage du $\operatorname{tsar}^{80}$ et les journaux des contemporains attentifs à la visite du tsar, tels que le marquis de Dangeau ${ }^{81}$.

Les informations du Nouveau Mercure (et, dans une moindre mesure, du journal Amsterdam), donnent au lecteur une image précise du tsar Pierre le Grand: l'image d'un monarque peu conventionnel. Il préfère être logé sans prétention, comme un soldat; il s'habille simplement et n'aime pas les cérémonies luxueuses et la curiosité de la foule ; il enfreint parfois les règles de l'étiquette et des comportements sociaux. Il a des goûts très inhabituels pour les Français. Ces caractéristiques pourraient être interprétées comme des preuves de la «barbarie» du tsar moscovite, ce que (contrairement aux auteurs de Mémoires !), les journalistes ne peuvent écrire directement. Mais, par ailleurs, le tsar fait preuve d'une inhabituelle soif de savoir. Il s'entretient avec les scientifiques et les professeurs en montrant des connaissances exceptionnelles dans les sciences les plus diverses - de la médecine à l'astronomie et la connaissance des langues étrangères. Quand il visite les résidences royales, il n'est pas seulement attiré par les divertissements et les festins (quoiqu'il n'y soit pas étranger), mais aussi et surtout par l'architecture, l'art du paysage, les techniques. Il manifeste du goût pour la peinture, pour l'art de la sculpture et des médailles, pour les théories artistiques. Il est difficile de dire pour autant que les journaux donnent de Pierre le Grand l'image d'un monarque éclairé, car les rapports sur ses activités d'Etat sont, en soi, très brèves. Cependant, ces périodiques ont donné une impulsion à la création de cette image. En effet, le portrait du tsar que peint cette presse est loin d'être celui d'un « maniaque sanglant, coupeur de barbes et de têtes", que Lortholary prétend, à tort, être celui qui était majoritairement diffusé en France vers $1717^{82}$. La représentation de l'activité du tsar dans cette presse contribue ainsi à détruire l'image négative de la Russie qui s'est constituée en France au cours du XVII ${ }^{\mathrm{e}}$ siècle $^{83}$.

Après une longue période de mépris envers ces contrées «barbares » et lointaines, on voit ainsi apparaître en France de l'intérêt pour Pierre $\mathrm{I}^{\mathrm{er}}$ et pour la Russie. Dans une récente

\footnotetext{
${ }^{80}$ Гистория Свейской войны, р. 610-625.

${ }^{81}$ Journal du marquis de Dangeau. Publié en entier pour la première fois par MM. Soulié, Dussieux, de Chennevières, Mantz, de Montaiglon; avec les additions inédites du duc de Saint-Simon publiées par M. Feuillet de Conches (Paris : Firmin-Didot frères, fils et $C^{\text {ie }}, 1859$ ), t. 1-19, XVII : 1717-1719, p. 77-114.

${ }^{82}$ Albert Lortholary, Les «philosophes » du XVIII siècle et la Russie, p. 20.

${ }^{83}$ Voir Michel Mervaud et Jean-Claude Roberti, Une infinie brutalité. L'image de la Russie dans la France des XVI et XVII siècles, Paris : IMSECO-Institut d'études slaves, 1991.
} 
publication, un historien fait ainsi remarquer que «Pierre <...> a finalement gagné sa guerre contre les préjugés anti-russes ${ }^{84}$. Le tsar a maintenant ses partisans dans les couches cultivées en France: Fontenelle, Saint-Simon, Voltaire se mettent à écrire le mythe de «créateur d'une nouvelle nation » qui arrache son peuple à la "barbarie ». Grâce à des

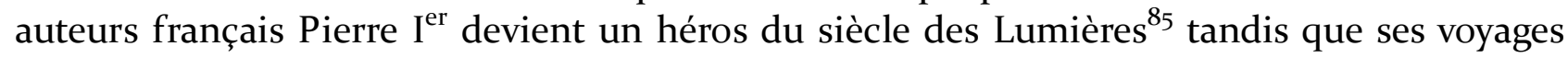
européens symbolisent l'initiation de la Russie à la « civilisation ».

\footnotetext{
${ }^{84} \mathrm{Ph}$. Champion, Les soixante-six jours français du tsar Pierre I-er: Avril-juin 1717, Paris : Le Monde de l'art \& des lettres ; C.E.R.D, 2004, p. 34.

${ }^{85}$ Voir : C. Мезин, Взгляд из Европы: французские авторы XVIII века о Петре I [Sergueï Mézine, Regard de l'Europe : les auteurs français du XVIII' siècle sur Pierre I ${ }^{e r}$ ] Saratov : izd. Saratovskogo ouniversiteta, 2003.
} 